\title{
Crowdsourcing In-Situ Data on Land Cover and Land Use Using Gamification and Mobile Technology
}

\author{
Juan Carlos Laso Bayas ${ }^{1, *}$, Linda See ${ }^{1}$, Steffen Fritz ${ }^{1}$, Tobias Sturn ${ }^{1}$, Christoph Perger ${ }^{1}$, \\ Martina Dürauer ${ }^{1}$, Mathias Karner ${ }^{1}$, Inian Moorthy ${ }^{1}$, Dmitry Schepaschenko ${ }^{1,2}$, \\ Dahlia Domian ${ }^{1}$ and Ian McCallum ${ }^{1}$ \\ 1 Ecosystems Services and Management Program, International Institute for Applied Systems Analysis, \\ Laxenburg A-2361, Austria; see@iiasa.ac.at (L.S.); fritz@iiasa.ac.at (S.F.); sturn@iiasa.ac.at (T.S.); \\ pergerch@iiasa.ac.at (C.P.); duerauer@iiasa.ac.at (M.D.); karner@iiasa.ac.at (M.K.); moorthy@iiasa.ac.at (I.M.); \\ schepd@iiasa.ac.at (D.S.); domian@iiasa.ac.at (D.D.); mccallum@iiasa.ac.at (I.M.) \\ 2 Forestry Faculty, Bauman Moscow State Technical University, Mytischi 141005, Russia \\ * Correspondence: lasobaya@iiasa.ac.at; Tel.: +43-2236-807-374
}

Academic Editors: Norman Kerle and Prasad S. Thenkabail

Received: 12 August 2016; Accepted: 26 October 2016; Published: 1 November 2016

\begin{abstract}
Citizens are increasingly becoming involved in data collection, whether for scientific purposes, to carry out micro-tasks, or as part of a gamified, competitive application. In some cases, volunteered data collection overlaps with that of mapping agencies, e.g., the citizen-based mapping of features in OpenStreetMap. LUCAS (Land Use Cover Area frame Sample) is one source of authoritative in-situ data that are collected every three years across EU member countries by trained personnel at a considerable cost to taxpayers. This paper presents a mobile application called FotoQuest Austria, which involves citizens in the crowdsourcing of in-situ land cover and land use data, including at locations of LUCAS sample points in Austria. The results from a campaign run during the summer of 2015 suggest that land cover and land use can be crowdsourced using a simple protocol based on LUCAS. This has implications for remote sensing as this data stream represents a new source of potentially valuable information for the training and validation of land cover maps as well as for area estimation purposes. Although the most detailed and challenging classes were more difficult for untrained citizens to recognize, the agreement between the crowdsourced data and the LUCAS data for basic high level land cover and land use classes in homogeneous areas (ca. $80 \%$ ) shows clear potential. Recommendations for how to further improve the quality of the crowdsourced data in the context of LUCAS are provided so that this source of data might one day be accurate enough for land cover mapping purposes.
\end{abstract}

Keywords: crowdsourcing; citizen science; volunteered geographic information; photocaching; LUCAS; land cover; land use; gamification; mobile phones

\section{Introduction}

Members of the public, acting as non-expert volunteers, have been involved in scientific research for more than a century [1] yet the rise of citizen science is a much more recent phenomenon [2]. At the same time, other initiatives that involve citizens have emerged, which are better described using the term crowdsourcing [3]. This is defined as the outsourcing of micro-tasks to the public, either for small payments using a platform such as Amazon's Mechanical Turk [4], or for other incentives, e.g., helping to find the missing Malaysian airlines plane (MH370) by collectively examining very high resolution satellite imagery over a vast geographical area [5]. This rise in the active involvement of the public can be attributed to a number of factors including the proliferation of location-enabled mobile devices (which has also led to the term Volunteered Geographic Information (VGI), where citizens are sensors 
of location-based information [6]); access to global, very high resolution satellite imagery via Google and Bing; and the development of Web 2.0 technology, which has led to a whole new generation of user-generated content including online mapping and neogeography [7].

The majority of citizen science applications can be found in the field of ecology, biodiversity and nature conservation [8], primarily due to the long history of citizen involvement in these domains. Amateur weather stations are another source of data that have been contributed by citizens for decades [9]. More recent applications include OpenStreetMap (OSM), which is a hugely successful collaborative online mapping initiative [10], volunteer efforts in disaster response [11,12] and environmental monitoring [13,14], among others (e.g., see [8]). However, an area where there is still considerable unrealized potential for crowdsourcing and citizen science is in the collection of in-situ data for the calibration and validation of products derived from Earth Observation (EO) [15]. Recent examples include Geo-Wiki and VIEW-IT for the interpretation of very high resolution satellite imagery by volunteers for land cover applications [16-18] and the iSpex mobile app for validation of in-situ air quality monitoring data and comparison with EO products on aerosol optical thickness, e.g., from MODIS [19]. There are many other examples of initiatives in which EO-relevant in-situ data are being crowdsourced but which are not yet being exploited for calibration or validation of EO-derived products [15].

One source of authoritative in-situ data is LUCAS (Land Use Cover Area frame Sample) [20], which is used for land cover and land use change detection in EU member countries, among many other applications [21]. In fact, LUCAS represents the only official in-situ dataset available for EU wide validation exercises such as the validation of the European Environment Agency's (EEA) very high resolution layers and CORINE land cover [22-24]. The LUCAS survey, which takes place every three years, is conducted in two stages. In the first stage, a systematic sample with a spacing of $2 \mathrm{~km}$ is laid on top of EU member countries, resulting in more than one million points. These are photo-interpreted and then placed into one of seven classes: arable land, permanent crops, grassland, wooded areas, shrubland, bareland, artificial land and water. From this, a smaller sample is drawn and each site is visited by a field surveyor unless the point is above $1500 \mathrm{~m}$ or inaccessible. This threshold was originally set at $1000 \mathrm{~m}$ in the 2006 and 2009 LUCAS surveys but was changed to $1500 \mathrm{~m}$ in the 2012 survey [25].

In 2015, there were 273,401 samples surveyed by 750 surveyors. Each surveyor followed a published set of protocols for data collection at each sample point [20]. The simplest part of the protocol is as follows: each point is photographed and then additional photographs are taken in the four cardinal directions away from the point. In addition, the surveyor notes down the land cover and land use from a very detailed, hierarchical classification [26]. There are several additional tasks associated with the survey protocols, e.g., walking a transect in an easterly direction while noting down changes in land cover and land use, and taking soil samples at certain locations.

If we use only the simple protocol for data collection, then we can consider the following practical research question: can citizens use a mobile application to collect data that could complement and enrich the LUCAS dataset and also provide an additional source of in-situ data for calibration and validation of remotely sensed products or for area estimation? This could be data that are either more densely sampled in a given area or collected more frequently than the LUCAS survey, which takes place every three years, or both. To answer this question, a mobile app called FotoQuest Austria was developed, and a data collection campaign was launched during the summer of 2015. The app is loosely based on the idea of geocaching, but rather than finding treasures at specific locations, players gain points for reaching a location, taking photographs, and documenting the land cover and land use. The app has a serious purpose but includes elements of gamification (i.e., the addition of game elements to existing applications [27]) to help motivate the volunteers. FotoQuest Austria also has similarities to the very recently released Pokémon GO app, which is played outdoors and also leads players to specific locations to find Pokémon. Gamification has been utilized to help motivate participation in citizen science applications; e.g., the Biotracker app, for contributing phenology data to Project Budburst, has been shown to attract a younger technologically-savvy audience (termed the 
"Millennials") by coupling technology with competitive elements such as badges and leaderboards [28]. The Cropland Capture game has also been successful in attracting players, which have collectively gathered more than 4.5 million classifications on the presence or absence of cropland from very high resolution satellite images and geo-tagged photographs [29,30].

The aim of this paper is to compare the results obtained from an application on in-situ crowdsourced data collection against LUCAS results in Austria. The paper is organized as follows. Section 2 provides an overview of the LUCAS land cover and land use classification, the FotoQuest Austria app and the campaign that was run to collect the data. The data are then described along with the methods by which the data were analyzed. Although the results show reasonable agreement at the highest and most general level of the LUCAS classification, we offer recommendations for how we can further improve the quality of land cover and land use data collected by citizens in the context of LUCAS, including use of the technology to enhance the data collection process in future, planned campaigns.

\section{LUCAS and FotoQuest Austria}

\subsection{LUCAS (Land Use Cover Area frame Sample)}

As mentioned in the introduction, LUCAS is an in-situ sample of land use and land cover carried out across EU member countries every three years [31]. The first LUCAS exercise was undertaken in 2006 and the most recent one was completed in 2015; the data from the 2015 survey were released at the end of July 2016. In Austria, there were 8844 LUCAS sample points visited in the 2015 survey. Using the protocol outlined in [20], surveyors classify the land cover and the land use at the sample points according to the LUCAS classification system, which is hierarchical, as outlined below.

Land cover has three levels in the hierarchy, where levels 1 and 2 are shown in Table 1 . There are 8 classes in level 1, which are then further expanded into multiple classes in level 2. Level 3 classes are the most detailed and the reader is referred to [26] for the complete list. For example, the level 2 class A10, which refers to Built-up areas, has three further sub-classes at level 3: A11 Buildings with one to three floors; A12 Buildings with more than three floors; and A13 Greenhouses. Similarly, for the Cropland class, level 3 goes down to the detail of individual crop types while the level 3 Forest classes identify broad tree species. In total, there are 77 land cover classes in the 2015 LUCAS classification.

Table 1. LUCAS (Land Use Cover Area frame Sample) land cover classification scheme.

\begin{tabular}{ccl}
\hline Level 1 & Description & \multicolumn{1}{c}{ Level 2 } \\
\hline A & Artificial Land & A10 (Built-up areas), A20 (Artificial non-built up areas) \\
\hline B & Cropland & B10 (Cereals), B20 (Root crops), ..., B80 (Other permanent crops) \\
\hline C & Woodland & $\begin{array}{l}\text { C10 (Broadleaved woodland), C20 (Coniferous woodland), } \\
\text { C30 (Mixed woodland) }\end{array}$ \\
\hline D & Shrubland & D10 (Shrubland with sparse tree cover), D20 (Shrubland w/o tree cover) \\
\hline E & Grassland & $\begin{array}{l}\text { E10 (Grassland with sparse tree/shrub cover) } \\
\text { E20 (Grassland without tree/shrub cover) }\end{array}$ \\
\hline F & Bare Land & $\begin{array}{l}\text { F10 (Rocks and stones), F20 (Sand), F30 (Lichens and moss), } \\
\text { F40 (Other bare soil) }\end{array}$ \\
\hline G & Water Areas & G10 (Inland water bodies), G20(Inland running water) \\
G30 (Coastal water bodies), G40 (Glaciers, permanent snow)
\end{tabular}

Land use has two levels in the hierarchy, where level 1 is shown in Table 2. For the Agriculture land use (U110), level 2 includes Production (U111), Fallow land (U112), and Kitchen garden (U113). Details of all level 2 land use classes can be found in [26]. In total, there are 33 distinct land use classes in the 2015 LUCAS classification. 
Table 2. LUCAS land use classification scheme.

\begin{tabular}{cc}
\hline Level 1 & Description \\
\hline U110 & Agriculture \\
U120 & Forestry \\
U130 & Aquaculture and Fishing \\
U140 & Mining and Quarrying \\
U210 & Energy Production \\
U220 & Industry and Manufacturing \\
U310 & Transport, Communications Networks, Storage and Protective Works \\
U320 & Water and Waste Treatment \\
U330 & Construction \\
U340 & Commerce, Finance, Business \\
U350 & Community Services \\
U360 & Recreation, Leisure, Sport \\
U370 & Residential \\
U400 & Unused and Abandoned Areas \\
\hline
\end{tabular}

LUCAS data are used for many different purposes including urban planning, monitoring of biodiversity and nature protection, monitoring of climate change; the management of forests, and protection of soils and agricultural policy, among other uses [31], as well as validation of Copernicus land cover products [24]. A visual browser for LUCAS has been built [32], which allows users to view data from different sources including LUCAS photographs to help interpret satellite imagery better. The browser can also be used to query locations for specific land cover and land use types or where change between surveys has occurred.

\subsection{The FotoQuest Austria Mobile App}

FotoQuest Austria was developed as a mobile game application for both the iOS and Android operating systems for the purpose of in-situ data collection following the LUCAS protocol. The app was developed to fit into the Geo-Wiki environment and makes use of the platform Ajax-API (Asynchronous Javascript and XML-Application Programming Interface). For web visualization, a Web Map Service (WMS) is used through MapServer. An open source database system (PostgreSQL) including PostGIS is used for storing and managing spatial information.

Figure 1 provides some screenshots to illustrate how the app works. When the app is first loaded, it shows the player a map of the surrounding area, their location on the map and the locations of quests in yellow and blue. Blue means that the location has not been visited yet and is worth 100 points while yellow denotes visits by up to 5 people. If a location has been visited by more than 5 people, it will be shown in red. Each time that a location is visited, the number of points that a player receives decreases, which is intended to incentivize players to seek out points that have not been visited before or less frequently.

When a player is within $50 \mathrm{~m}$ of a point, the option to begin the quest will be active (Figure 1c). The player will then be asked if the point is reachable or not, and if not reachable, then whether it is visible or not. If the point is visible, players will then be asked to take one photograph in the direction of the point and the quest will be complete. Otherwise, they will be told to move closer to the point. Once they are close enough, the first part involves taking photographs in four directions: North, East, South and West. The app uses the compass to guide players and only allows the photographs to be taken when the mobile phone is pointed in the correct direction. For example, Figure 2a shows the instruction to take a picture in the northerly direction. The button for taking the photograph will only turn green if the compass indicates north. Figure $2 b$ shows Step 5 in the sequence of photographs that players are instructed to take, which is at the actual point location itself. 


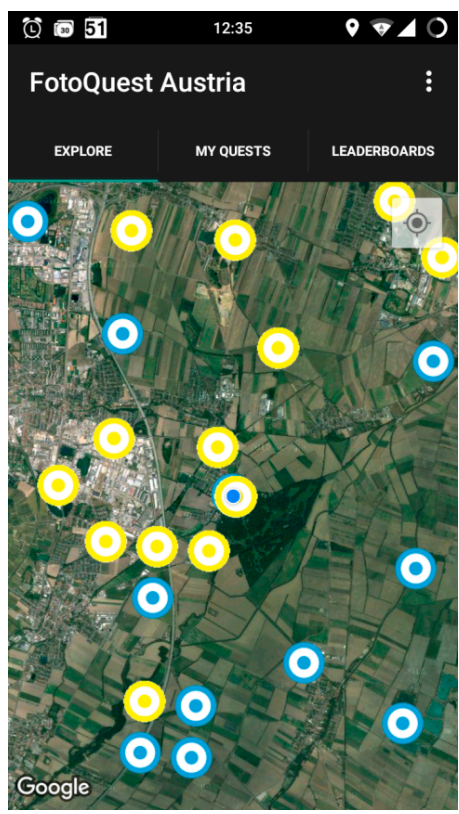

(a)

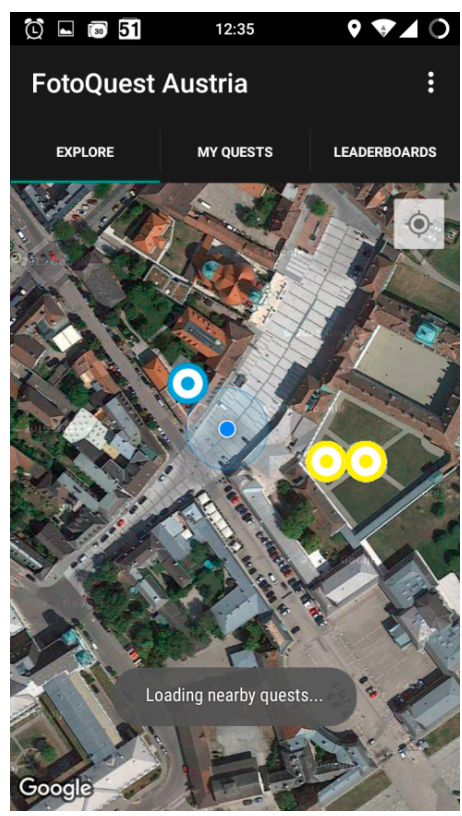

(b)

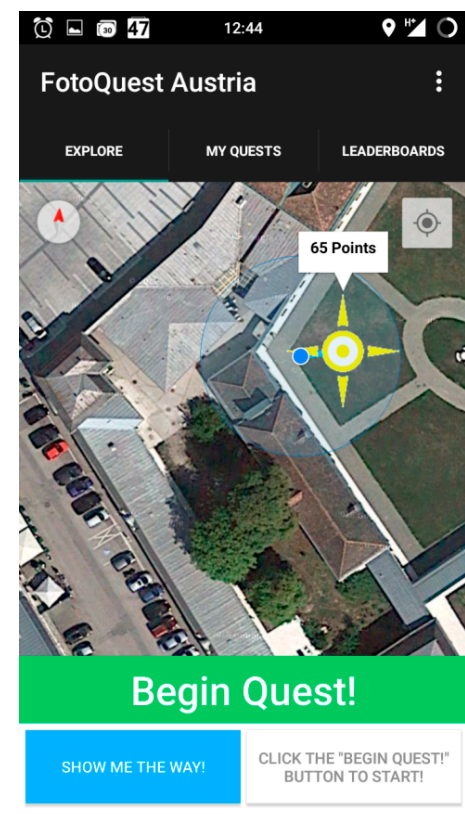

(c)

Figure 1. Screenshots from the FotoQuest Austria app showing: (a) the location of available quests in the surrounding area; (b) the available quests in the more immediate area; and (c) a nearby quest with the value in points that a player can achieve if the quest is successfully undertaken. Blue dots denote locations that have not yet been visited while yellow means that the quest has been undertaken between one and five times already.

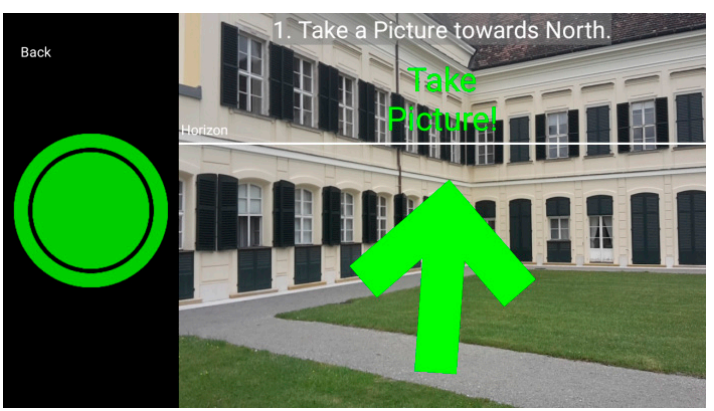

(a)
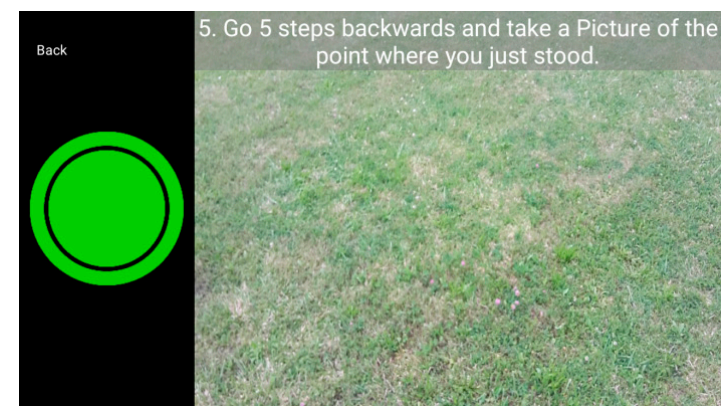

(b)

Figure 2. Screenshots from the FotoQuest Austria app showing: (a) Step 1, in which players are asked to take a picture in a northerly direction; and (b) Step 5, in which players take a picture of the actual point.

A guiding line is provided for players to line up the mobile phone with the horizon so that one third of the photograph contains sky and two thirds landscape (where relevant). The player then takes pictures in the remaining cardinal directions, and in step five, the player is instructed to take a picture of the actual point location. After the photographs are taken, the player is then presented with menu options for classifying the land cover as shown in Figure 3a-c. 


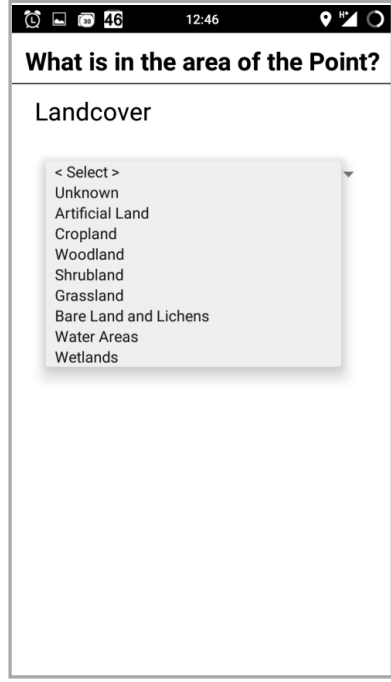

(a)

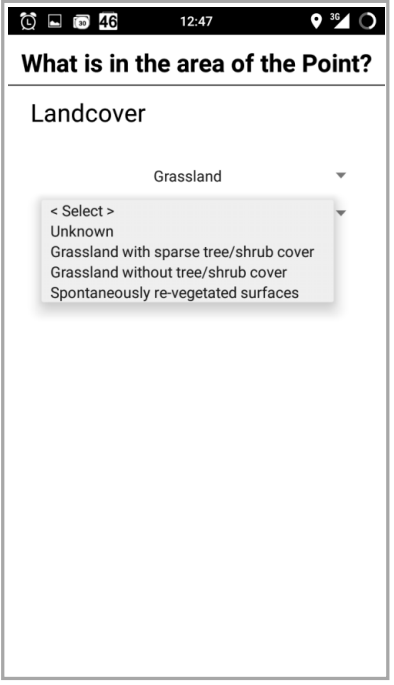

(b)

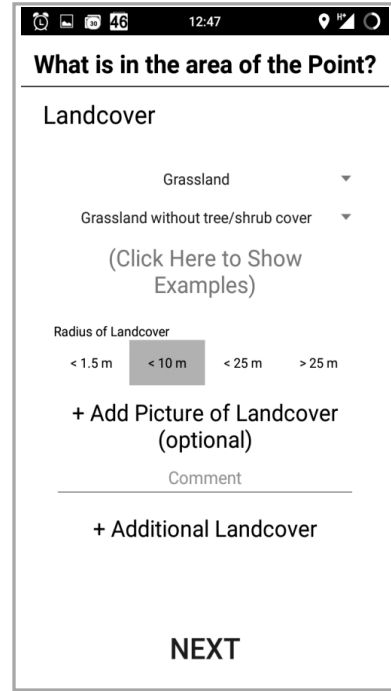

(c)

Figure 3. Screenshots from the FotoQuest Austria app showing: (a) land cover choices at level 1; (b) land cover choices at level 2, which are dependent upon the choice made at level 1; and (c) the radius of homogeneous land cover, with the option to add a picture.

Figure 3a shows land cover choices from level 1 of the LUCAS classification scheme. Once the player chooses a level 1 land cover, they are shown choices at level 2 (Figure 3b) and level 3 (if relevant). Once all the land cover choices are made, the player is asked to select a radius (Figure 3c), which provides an indication of how homogeneous the land cover is around the point location. The choices are $<1.5 \mathrm{~m}$ for points that are very heterogeneous and $>25 \mathrm{~m}$ for very homogenous points, with two choices in between, i.e., $<10 \mathrm{~m}$ and $<25 \mathrm{~m}$. Finally, the player can take an optional photograph and select additional land cover types for very heterogeneous landscapes. The player is then provided with similar screens but for land use, again following the LUCAS hierarchical nomenclature. Once the land cover and land use choices are made, the player is asked whether they want to upload the photographs. The quest is then finished, players are awarded points for the quest and they can continue exploring other locations indicated on the map. No specific training was done with the players regarding land use or land cover recognition, e.g., we did not provide pictures of typical land use or land cover in the app. Since then, we have added example photographs of different land cover and land use types and we are currently working on a decision tree to help players decide on the land use/land cover. The app was nevertheless promoted through the Geo-Wiki Facebook page and twitter account where users could directly contact the team with any questions.

\subsection{The FotoQuest Austria Data Collection Campaign}

The FotoQuest Austria app was launched in Austria during the summer of 2015 via a media campaign, i.e., a press release was issued and interviews were held with the main television and radio stations in Austria. The app was featured as "app of the week" in the technology section of Austria's main TV channel website and was featured on an afternoon program which demonstrated how the app worked.

For each quest, the photographs were stored and linked to the data collected. In addition to the player, location information, and the data pertaining to the quest itself, additional data of interest were recorded including the type of device used and whether the location corresponds to a LUCAS sample point or not. Many more locations were added to the game beyond the LUCAS sample points so that more quests were available for the players, particularly in urban areas.

In total, there were 2234 quests made at 1699 unique locations, and 12,278 geo-tagged photographs were collected. Just under $30 \%$ of the quests were made using an iPhone while the majority $(>70 \%)$ were made using an Android phone. The campaign ran until the end of September 2015 and prizes 
were awarded to the top scoring players, which included a smartphone and some tablets. Some people have continued to play the game so the data used in this paper include points collected until the end of December 2015.

\section{Materials and Methods}

\subsection{Data Pre-Processing}

In order to compare data collected by LUCAS with that from FotoQuest Austria, the first task was to select data corresponding to those quests that coincide with LUCAS 2015 points. The result was 677 overlapping points. Of these, more than $50 \%$ were subsequently excluded due to reasons such as being taken before the start date of the campaign, points that were reported as being not visible, and land use or land cover that was reported as unknown, leaving a total of 306 points in common.

Figure 4 shows the location of LUCAS 2015 and FotoQuest Austria points, highlighting those that were used in the analysis. The points shown in green in Figure 4 are the LUCAS points, of which there are 8844 in total. For the FotoQuest campaign, additional points were then added, densifying the sample, particularly in urban areas (to target urban sprawl) and in wetland areas, which are currently not well mapped in Austria. The total number of unique locations or quests available across Austria was 62,894 or roughly 8 times more than the available LUCAS points. Hence, the LUCAS points are only a sub-sample of the total number of points in the game, which shows how much more in-situ data would potentially be available for calibration and validation of remotely-sensed products if all the locations were visited. The red points shown in Figure 4 are those that were collected during the FotoQuest Austria campaign while the yellow ones are those that were used in the analysis once some of the data were excluded, as described above.

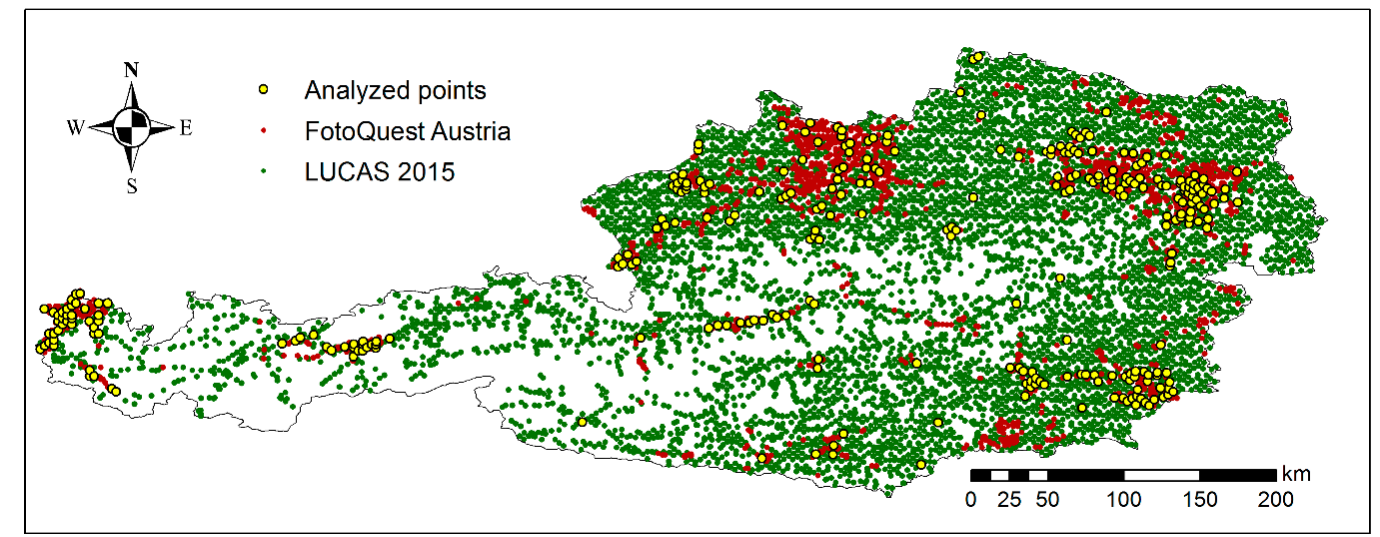

Figure 4. Overlay of FotoQuest Austria and LUCAS 2015 points used in the analysis. Red points are the points visited during the FotoQuest Austria campaign. A large swath of points $(N=371)$ was not considered in the analysis due to the reasons listed in Section 3.1.

The database from FotoQuest Austria had one row per entry for each land use or land cover, with all the information from a given location spanning multiple rows. These could come from the same player, describing all land uses and land cover types seen within a radius from the point, but also from several players evaluating the same point. A summary of all the entries per point was done, and this summary statistic was then added to the main database. An agreement response variable was added to reflect agreement between the answers provided for land cover and land use at each location compared to the land cover and land use recorded by the LUCAS surveyor. Agreement was separated into three levels for land cover and two levels for land use, which corresponds to the LUCAS classification system, arranged into a hierarchy as described in Section 2.1. Thus, there could be agreement at the highest level of land cover or land use (i.e., level 1) or at higher levels (levels 2 and 3) based on how detailed an answer was provided by each player and at each point. 


\subsection{Statistical Analysis}

Confusion matrices between the authoritative LUCAS data and the answers provided by the players were tabulated in order to calculate the overall agreement for land cover and land use at each of the levels of the LUCAS classification and class agreement at level 1. Another important consideration was to understand if the agreement levels differed when the points visited were in areas with a mixture of land cover or land use. For this, a visual inspection of the LUCAS survey pictures and a $20 \mathrm{~m}$ radius of the relevant point on satellite imagery from Google Earth was undertaken to identify homogeneous points as shown in Figure 5. Homogeneous points (hg, Figure 5a) were those in which all of the photographs and the Google Earth imagery showed the same land cover; potentially homogeneous (pt) is when 2 to 3 of the photographs showed the same land cover but the photographs were taken from a road while heterogeneous (ht, Figure $5 b$ ) means different land cover types shown in the photographs. Overall agreement was calculated separately on these three degrees of homogeneity to see whether this had an influence on the performance of the players.

A set of statistical models was then derived to test the agreement between the land cover and land use data from the players in relation to the authoritative LUCAS data, i.e., the response variables, using several possible predictors of the agreement differences. These include the variables radius (RD), which represents the visible distance for a given land use or land cover in meters (4 categories) around the point in FotoQuest, the distance between the actual point and the LUCAS point coordinates (D) and the three levels of homogeneity (HM), i.e., homogeneous, potentially homogeneous and heterogeneous (hg, pt, and ht, respectively). Additionally, since each point could be evaluated several times by different players and each player could have evaluated several points, the total number of observations that each player made (OBSU) as well as the total number of times each point was visited (OBPT) were included as covariates. From a total of 76 players, all visited 25 points on average with the exception of one player who visited 83 points.

Furthermore, random effects for the player and unique point identifications (PLAYER-ID, POINT-ID) were included to deal with lack of independence issues, i.e., several observations made by the same player and several evaluations per point.

Once the predictors were selected, generalized linear mixed models with binomial responses were developed to analyze the agreement at each of the three levels of land cover and two levels of land use. The Laplace estimation method was employed to fit the models using Proc Glimmix, SAS v9.4. Random effects were evaluated using statistical inference for the covariance parameters (significance test based on the ratio of residual likelihoods) via the inclusion of a "covtest glm" statement and the comparison of relative goodness of fit of models with and without these effects. The Akaike Information Criterion (AIC) [33] reported by the software was used to compare the relative goodness of fit. A general representation of the fixed effects model is given by:

$$
\mathrm{Y}=f(\mathrm{D}, \mathrm{RD}, \mathrm{HM}, \mathrm{OBSU}, \mathrm{OBPT}:: \text { PLAYER-ID, POINT-ID) }
$$

where $\mathrm{Y}=$ binomial response indicating overall agreement of FotoQuest Austria data with LUCAS 2015 (yes $=1$, no $=0$ ). The double colon separates fixed and random effects.

Since the actual point coordinates were seldom reported in the FotoQuest dataset, observations with values for $\mathrm{D}$ were very limited (less than $25 \%$ ). A univariate model with $\mathrm{D}$ as the only predictor was run but no significant effect was found $(p>0.05)$. D was consequently excluded from the final multivariate model, which is provided in Equation (2) as:

$$
\mathrm{Y}=f(\mathrm{RD}, \mathrm{HM}, \mathrm{OBSU}, \mathrm{OBPT}:: \text { PLAYER-ID, POINT-ID) }
$$

Moreover, there was one player who visited 83 different points and one location was visited 15 times as shown in Figure 6. Thus, these extreme observations were also eliminated before fitting the models. 


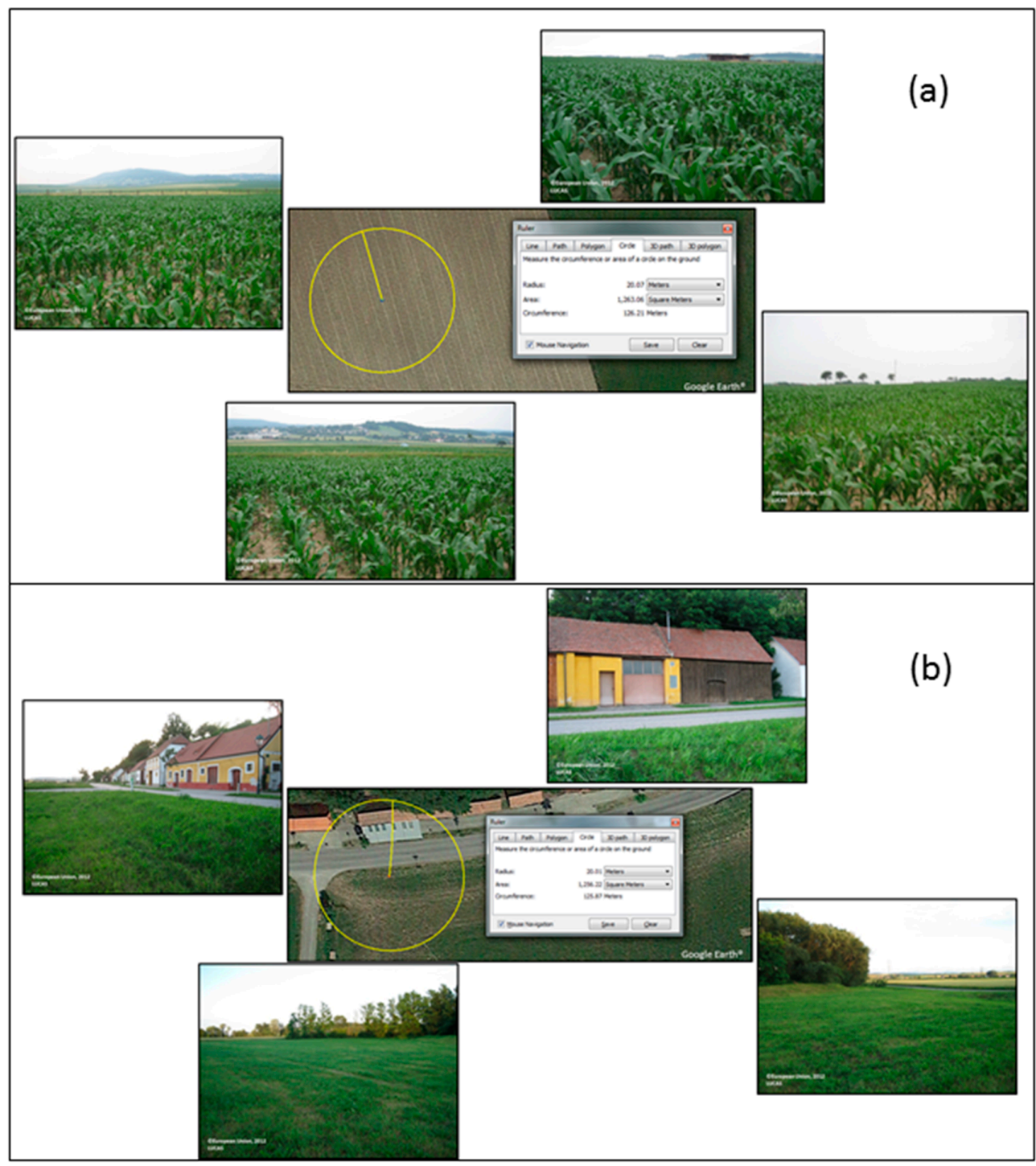

Figure 5. Examples of points with: (a) homogeneous; and (b) heterogeneous/mixed landscapes as determined by inspecting the LUCAS pictures and Google Earth ${ }^{\circledR}$ satellite imagery. The circle radius is approximately 20 meters around the point.
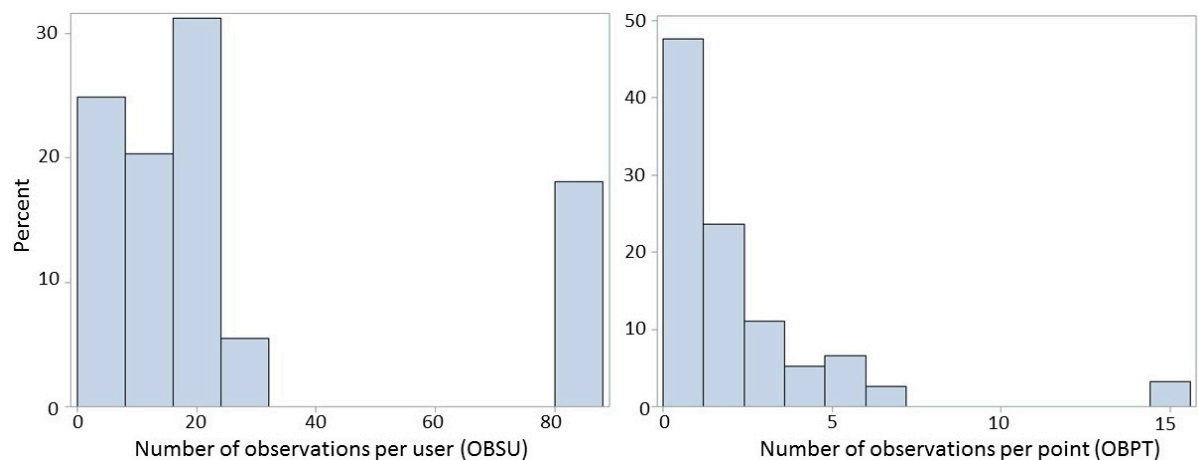

Figure 6. Histograms showing the data distribution in percent, highlighting outliers in the number of observations per point (OBPT) and per player (OBSU).

Initial correlation analyses were run between the predictors before entering them in the models in order to control for collinearity. If collinearity was found, the decision on which predictor to choose 
was made using the Akaike Information Criterion (AIC) as a relative goodness of fit to compare between models with different predictor variables.

Finally, to understand if the power player influenced the agreement with LUCAS, a set of models for all levels of land use and land cover were run but only with a covariate (GR), which identifies whether the player was a power player (PP) (i.e., the person who visited 83 different points) or a regular player (RP). The simplified model is shown in Equation (3):

$$
\mathrm{Y}=f(\mathrm{GR}:: \text { PLAYER-ID, POINT-ID) }
$$

The results derived from these models (Equation (3)) are purely demonstrative because there was only one power player and therefore all observations coming from this power group are not independent. The results are merely provided to illustrate the differences between the two groups. Most of the models did not converge when POINT-ID was introduced as a random effect due to the low number of observations. The variable PLAYER-ID was evaluated at all times as a random effect.

\section{Results}

Table 3 shows the agreement values for three different sets of points: overall agreement, agreement at homogeneous points (hg), and agreement at not homogeneous points (pt and hg) for all levels of land cover and land use while the confusion matrices appear in Tables 4 and 5 . The overall agreement for land cover and land use at level 1 is similar, i.e., $68 \%-69 \%$, indicating reasonable performance by the players. For land use at level 2, the agreement drops to $62 \%$ while levels 2 and 3 for land cover are much lower, i.e., between $37 \%$ and $23 \%$. This indicates that more detailed land use is easier to identify than detailed land cover, which would require, e.g., tree type and crop type identification skills, which many people may not possess. Additionally, clear differences between homogeneous and more heterogeneous points can be observed, where homogeneous points always have higher agreement, ranging from $8 \%$ to $30 \%$ more.

Table 3. Agreement between LUCAS 2015 and FotoQuest in land cover (LC) and land use (LU) for three groups of points: Overall agreement, agreement at homogeneous points, and agreement at not homogeneous points in percentage. Number of points for each group $(N)$ is shown.

\begin{tabular}{cccccccc}
\hline \multirow{2}{*}{ Type } & \multirow{2}{*}{ Level } & \multicolumn{2}{c}{ Overall } & \multicolumn{2}{c}{ Homogeneous } & \multicolumn{2}{c}{ Not Homogeneous } \\
\cline { 2 - 7 } & & $\boldsymbol{N}$ & Mean (\%) & $\boldsymbol{N}$ & Mean (\%) & $\boldsymbol{N}$ & Mean (\%) \\
\hline \multirow{3}{*}{ LC } & 1 & 302 & 69 & 150 & 79 & 152 & 58 \\
& 2 & 282 & 37 & 138 & 46 & 144 & 28 \\
& 3 & 194 & 23 & 86 & 27 & 108 & 19 \\
\hline \multirow{2}{*}{ LU } & 1 & 304 & 68 & 150 & 81 & 154 & 56 \\
& 2 & 174 & 62 & 92 & 76 & 82 & 46 \\
\hline
\end{tabular}

In the confusion matrices, the results show that artificial land is sometimes confused with Grassland or Woodland or vice versa while Cropland is confused with Grassland at times (Table 4). For land use, there is some confusion between Agriculture, Residential and Transport classes (Table 5).

Table 4. Confusion matrix showing level 1 land cover classifications from FotoQuest and LUCAS 2015.

\begin{tabular}{cccccccccc}
\hline & Artificial & Cropland & Woodland & Shrubland Grassland & Bare Land & Water & Wetlands & User Acc. \\
\hline Artificial & 83 & 8 & 17 & 4 & 39 & 1 & 2 & 0 \\
Cropland & 5 & 73 & 9 & 2 & 27 & 4 & 1 & 0 \\
Woodland & 4 & 7 & 44 & 6 & 9 & 0 & 2 & 0.54 \\
Shrubland & 5 & 4 & 6 & 3 & 2 & 0 & 2 & 0 \\
FotoQuest & Grassland & 10 & 15 & 13 & 3 & 60 & 1 & 4 & 0 \\
Bare land & 1 & 1 & 0 & 0 & 2 & 0 & 0 & 2 \\
Water & 0 & 3 & 1 & 0 & 2 & 0 & 6 & 0 \\
Wetlands & 2 & 0 & 0 & 0 & 2 & 0 & 0 & 0.14 \\
Prod. Acc. & 0.75 & 0.66 & 0.49 & 0.17 & 0.42 & 0.00 & 0.35 & 0.00 \\
\hline
\end{tabular}


Table 5. Confusion matrix showing level 1 land use classifications from FotoQuest and LUCAS 2015.

\begin{tabular}{|c|c|c|c|c|c|c|c|c|c|c|c|c|c|c|c|c|}
\hline & & U110 & U120 & U130 & U140 & U210 & U220 & U310 & U320 & U330 & U340 & U350 & U360 & U370 & U400 & User Acc. \\
\hline & U110 & 159 & 18 & 1 & 0 & 0 & 0 & 21 & 0 & 0 & 1 & 2 & 3 & 15 & 8 & 0.70 \\
\hline & U120 & 9 & 44 & 0 & 0 & 0 & 0 & 1 & 0 & 0 & 0 & 0 & 0 & 1 & 1 & 0.79 \\
\hline & U130 & 0 & 0 & 0 & 0 & 0 & 0 & 1 & 0 & 0 & 0 & 0 & 0 & 0 & 0 & 0.00 \\
\hline & U140 & 0 & 0 & 0 & 0 & 0 & 0 & 1 & 0 & 0 & 0 & 0 & 0 & 1 & 0 & 0.00 \\
\hline & U210 & 1 & 0 & 0 & 0 & 0 & 0 & 1 & 0 & 1 & 0 & 0 & 0 & 0 & 0 & 0.00 \\
\hline & U220 & 0 & 0 & 0 & 0 & 0 & 0 & 2 & 0 & 0 & 1 & 0 & 0 & 3 & 0 & 0.00 \\
\hline & U310 & 2 & 1 & 0 & 0 & 2 & 0 & 6 & 0 & 0 & 1 & 0 & 1 & 2 & 1 & 0.38 \\
\hline \multirow[t]{8}{*}{ FotoQuest } & U320 & 1 & 0 & 0 & 0 & 0 & 0 & 3 & 0 & 0 & 0 & 0 & 0 & 0 & 0 & 0.00 \\
\hline & U330 & 0 & 0 & 0 & 0 & 0 & 0 & 0 & 0 & 0 & 0 & 0 & 1 & 0 & 0 & 0.00 \\
\hline & U340 & 0 & 0 & 0 & 0 & 0 & 0 & 1 & 0 & 0 & 1 & 0 & 1 & 0 & 0 & 0.33 \\
\hline & U350 & 0 & 0 & 0 & 0 & 0 & 0 & 1 & 0 & 0 & 0 & 0 & 0 & 0 & 0 & 0.00 \\
\hline & U360 & 6 & 2 & 0 & 0 & 1 & 0 & 4 & 0 & 0 & 1 & 0 & 7 & 2 & 1 & 0.29 \\
\hline & U370 & 24 & 3 & 0 & 0 & 0 & 0 & 23 & 0 & 0 & 1 & 2 & 4 & 38 & 1 & 0.40 \\
\hline & U400 & 7 & 1 & 0 & 0 & 0 & 0 & 2 & 0 & 0 & 0 & 1 & 2 & 0 & 3 & 0.19 \\
\hline & Prod. Acc. & 0.76 & 0.64 & 0.00 & NA & 0.00 & NA & 0.09 & NA & 0.00 & 0.17 & 0.00 & 0.37 & 0.61 & 0.20 & \\
\hline
\end{tabular}

Notes: U110: Agriculture; U120: Forestry; U130: Aquaculture and Fishing; U140: Mining and Quarrying; U210: Energy Production; U220: Industry and Manufacturing; U310: Transport, Communications Networks, Storage and Protective Works; U320: Water and Waste Treatment; U330: Construction; U340: Commerce, Finance, Business; U350: Community Services; U360: Recreation, Leisure, Sport; U370: Residential; U400: Unused and Abandoned Areas. 
Tables 6 and 8 show the overall agreement at all levels for the main land cover and land use classes in LUCAS. The land cover agreement at level 1 is very high for the Artificial Land and Cropland classes, which together cover $44 \%$ of the points. The Grassland and Woodland classes have lower agreement, between $60 \%$ and $62 \%$, and represent almost $50 \%$ of the dataset. Although the agreement shown in these tables does not represent the exact agreement for individual sub-classes at lower levels, which also differ in number inside each main class (e.g., Tables 1 and 7), it does provide an overall idea of accordance. For the purpose of land cover mapping, these high level classes (level 1) are generally sufficient so we focus mainly on these.

Table 6. Land cover main agreement (Ag) areas (sorted by percentage covered in FotoQuest Austria) between the two systems. Number of observations $(n)$ is shown.

\begin{tabular}{ccccccccc}
\hline \multirow{2}{*}{ Type } & $\begin{array}{c}\text { Coverage in FotoQuest } \\
\text { Austria (\%) }\end{array}$ & \multicolumn{2}{c}{ Level 1 } & \multicolumn{2}{c}{ Level 2 } & \multicolumn{2}{c}{ Level 3 } \\
\cline { 3 - 8 } & & $\boldsymbol{n}$ & Ag (\%) & $\boldsymbol{n}$ & Ag (\%) & $\boldsymbol{n}$ & Ag (\%) \\
\hline Grassland & 29 & 143 & 62 & 138 & 33 & 99 & 0 \\
Cropland & 22 & 111 & 89 & 100 & 51 & 78 & 50 \\
Artificial Land & 22 & 110 & 90 & 109 & 39 & 100 & 21 \\
Woodland & 18 & 90 & 60 & 87 & 29 & 67 & 19 \\
Others & 9 & 43 & $0-76$ & 43 & $0-76$ & 29 & 0 \\
\hline
\end{tabular}

In Table 7, an example for the main class "Cropland" at level 3 is shown to illustrate the differences between the agreements that exist in different subclasses within a main class. Crops that are relatively easy to recognize, i.e., maize and wheat, have higher agreement than the others, which when averaged out result in around $50 \%$ agreement for this level 1 class.

Table 7. An example of land cover type agreement at level 3 for the main class "Cropland", sorted by percentage agreement between FotoQuest Austria and LUCAS. Number of observations $(n)$ is shown.

\begin{tabular}{ccc}
\hline Crop Type at Level 3, Cropland & $\boldsymbol{n}$ & Agreement (\%) \\
\hline Maize & 32 & 81 \\
Common Wheat & 5 & 80 \\
Clovers & 3 & 33 \\
Barley & 7 & 14 \\
Vineyards & 4 & 1 \\
Soya & 11 & 0 \\
Other root crops & 4 & 0 \\
Others & 12 & $0-1$ \\
\hline
\end{tabular}

Likewise, there is over $90 \%$ land use agreement in the Agriculture land use class and over $70 \%$ for the Residential and Forestry classes in level 1 (Table 8). The Transport land use class has an agreement of ca. $21 \%$ but represents only $15 \%$ of the points in the database. Other land use classes represent $10 \%$ of the sample and have agreements from $53 \%$ and lower.

Table 8. Land use main agreement (Ag) areas (sorted by percentage covered in FotoQuest Austria) between the two systems. Number of observations $(n)$ is shown.

\begin{tabular}{cccccc}
\hline \multirow{2}{*}{ Type } & $\begin{array}{c}\text { Coverage in FotoQuest } \\
\text { Austria (\%) }\end{array}$ & \multicolumn{2}{c}{ Level 1 } & \multicolumn{2}{c}{ Level 2 } \\
\cline { 3 - 6 } & & $\boldsymbol{n}$ & Ag (\%) & $\boldsymbol{n}$ & Ag (\%) \\
\hline Agriculture & 46 & 209 & 89 & 169 & 91 \\
Forestry & 15 & 69 & 74 & 21 & 0 \\
Transport plus & 15 & 67 & 21 & 44 & 23 \\
Residential & 14 & 63 & 73 & 25 & 0 \\
Others & 10 & 50 & $0-53$ & 38 & $0-40$ \\
\hline
\end{tabular}

${ }^{\dagger}$ Tertiary Sector, Transport, Utilities and Residential. 
In the land cover model at level 2 (Table 9), additionally to significant homogeneity (HM) effects (Table 10), the number of observations per user (OBSU) had a significant effect on agreement, with every additional observation increasing the chances of agreement between FotoQuest and LUCAS by up to $1 \%$ (regression coefficient (reg. coeff.) \pm standard error (std. err): $0.0055 \pm 0.0022$ ).

In the land use model at level 1, apart from significant effects from homogeneity and radius (Table 10), for every additional observation at a given point, the chances of land use agreement between the two systems increased by $32 \%$ (reg. coeff. \pm std. err.: $0.2739 \pm 0.1010$ ), which is in line with the findings in [34], i.e., the positional accuracy of road features in OpenStreetMap increased with an increase in the number of contributors. At level 2 in the land use model, significant effects of HM and RD were noted (Table 9), but also for every additional observation a given player has made, the chances of land use agreement between the two systems increased by $8 \%$ (reg. coeff. \pm std. err.: $0.0772 \pm 0.0265$ ).

Table 9. Models tested for agreement (yes/no) between FotoQuest Austria and LUCAS 2015 land cover (LC) and land use (LU). $p$-values are shown when appropriate. Number of observations ( $n$ ) is shown. ns $=$ not significant.

\begin{tabular}{ccccccccc}
\hline Type & Level & $\boldsymbol{n}$ & Players & Points & RD & HM & OBSU & OBPT \\
\hline \multirow{2}{*}{ LC } & 1 & 497 & 73 & 302 & ns & $<0.001$ & Ns & ns \\
& 2 & 477 & 72 & 282 & ns & ns & 0.013 & ns \\
& 3 & 373 & 60 & 194 & ns & ns & Ns & ns \\
\hline \multirow{2}{*}{ LU } & 1 & 458 & 76 & 304 & 0.005 & $<0.001$ & Ns & 0.007 \\
& 2 & 297 & 60 & 174 & $<0.001$ & $<0.001$ & 0.004 & ns \\
\hline
\end{tabular}

Notes: $\mathrm{RD}=$ radius; $\mathrm{HM}=$ homogeneity, GR = group (power player or not)/OBSU = number of observations per player; $\mathrm{OBPT}=$ number of visits per point.

Table 10. Differences between homogeneity (HM) levels, comparing homogeneous (hg), potentially homogeneous (pt) and heterogeneous (ht) points and the radius (RD) in relation to land cover (LC) and land use (LU) agreement (0-1) between FotoQuest Austria and LUCAS 2015. Adjusted means \pm standard errors are shown. Different letters indicate significant differences in a given level and predictor at a significance level of $\alpha=5 \%$.

\begin{tabular}{|c|c|c|c|c|c|c|c|c|}
\hline \multirow{2}{*}{ Type } & & \multicolumn{3}{|c|}{ HM } & \multicolumn{4}{|c|}{ RD } \\
\hline & & hg & pt & ht & 1 & 10 & 25 & 50 \\
\hline \multirow{3}{*}{$\mathrm{LC}$} & 1 & $0.82 \pm 0.04^{\mathrm{a}}$ & $0.66 \pm 0.07^{b}$ & $0.59 \pm 0.06^{b}$ & ns & ns & ns & ns \\
\hline & 2 & ns & ns & ns & ns & ns & ns & ns \\
\hline & 3 & ns & ns & ns & ns & ns & ns & ns \\
\hline \multirow{2}{*}{ LU } & 1 & $0.70 \pm 0.07^{\mathrm{A}}$ & $0.41 \pm 0.09^{\mathrm{B}}$ & $0.27 \pm 0.07^{\mathrm{B}}$ & $0.08 \pm 0.08^{b}$ & $0.58 \pm 0.09^{a}$ & $0.60 \pm 0.08^{a}$ & $0.74 \pm 0.03^{a}$ \\
\hline & 2 & $0.67 \pm 0.07^{\mathrm{a}}$ & $0.55 \pm 0.09^{a}$ & $0.16 \pm 0.05^{b}$ & + & $0.22 \pm 0.08^{\mathrm{B}}$ & $0.44 \pm 0.10^{\mathrm{B}}$ & $0.69 \pm 0.05^{\mathrm{A}}$ \\
\hline
\end{tabular}

On the one hand, there is an overall trend for homogeneous points (hg) to have significantly higher agreement in land use and land cover than heterogeneous points (ht) (HM, Table 10), with the exceptions of land cover level 2 and 3, where no variable was found to be significant. On the other hand, the trend observed for radius (RD, Table 10) shows that when the radius reported is large, the agreement in land use is much higher although in some levels there are no significant differences between some adjacent radii.

When comparing the agreement from the one power player with the agreement from all other players (Table 11), it appears that the power player always has higher agreement, although these differences were not significant, except for land cover level 2, where the power player had significantly higher agreement than the other players. These differences are due to the lower variability existing in this group. 
Table 11. Models tested to compare power player (PP) evaluations and regular players (RP) with regards to the agreement (yes/no) in land cover (LC) and land use (LU) between FotoQuest Austria and LUCAS 2015. Number of observations $(n)$ is shown.

\begin{tabular}{ccccc}
\hline Type & Level & $\boldsymbol{n}$ & $\begin{array}{c}\text { Adjusted Mean } \pm \text { Standard } \\
\text { Error Agreement RP (\%) }\end{array}$ & $\begin{array}{c}\text { Adjusted Mean } \pm \text { Standard } \\
\text { Error Agreement PP (\%) }\end{array}$ \\
\hline \multirow{2}{*}{ LC } & 1 & 497 & $68 \pm 3.2^{\mathrm{a}}$ & $85 \pm 8.0^{\mathrm{a}}$ \\
& 2 & 477 & $35 \pm 2.4^{\mathrm{B}}$ & $46 \pm 4.7^{\mathrm{A}}$ \\
& 3 & 373 & $12 \pm 3.3^{\mathrm{a}}$ & $27 \pm 21.1^{\mathrm{a}}$ \\
\hline \multirow{2}{*}{ LU } & 1 & 458 & $64 \pm 3.2^{\mathrm{A}}$ & $78 \pm 9.2^{\mathrm{A}}$ \\
& 2 & 297 & $51 \pm 5.4^{\mathrm{a}}$ & $64 \pm 21.5^{\mathrm{a}}$ \\
\hline \multicolumn{4}{r}{}
\end{tabular}

\section{Discussion}

The results of this first campaign with the FotoQuest Austria app show the willingness and potential of citizens to be involved in collecting data that are of relevance to both LUCAS and for developing training and validation datasets for Earth Observation data, especially when homogeneous points are considered. At present the accuracy is not sufficient for calibration and validation of remotely sensed products. Changes that are currently being implemented in the latest version of the app include improved training, e.g., videos showing the procedures to follow and images of typical land cover/land use as well as recognition of specific crops. We will also use control points where a known stable land cover/use exists and then tell the player whether their answer was correct and why as a form of corrective feedback in future versions. All these new features, together with in-app display of satellite imagery from the surrounding area and a decision tree to aid land cover/land use classification should help to obtain higher accuracies in the future. We will also consider the use of tools from Cognitive Task Analysis (CTA) as outlined in [35], in particular the use of a walk through, online questionnaires and semi-structured interviews with players as a way of better understanding how they perceive land cover and land use and as ways to improve the training materials and app more generally. At the highest level of the LUCAS classification for both land cover and land use, the overall agreement was ca. 70\% and in urban areas and cropland reached ca. $90 \%$. These two main classes are easy to recognize and do not require any training showing that at Level 1, players could perform well without additional training. Some improvements are needed in the recognition of these last two classes, to reduce the confusion between Grassland and Artificial, and for recognition of transport-related land uses. The former may be related to heterogeneity, e.g., are a few trees scattered in a grassy area considered to be Grassland or Woodland, while the latter may require more specialist knowledge. Regarding the confusion between Grassland and Artificial, which one would think should not occur, the issue may again be the heterogeneity of the landscape. When the point falls in a small grassland area (e.g., a park or large garden) which is located within a larger artificial setting or on a road next to a grassy area, the player may have been confused as to which land cover class to choose. From a land cover mapping perspective, the ability to recognize transport-related land uses is less relevant.

When the more detailed classification of land cover from FotoQuest Austria is compared with the authoritative LUCAS data (i.e., levels 2 and 3), the agreement is much lower and hence the performance by the crowd is relatively poor in comparison to level 1. Looking at these more detailed classes (Section 2.1), it is clear that some specialist knowledge or training is required, e.g., to be able to differentiate between different crop or forest types. For example, at level 3, the overall agreement for crop types was $50 \%$. However, common crop types such as maize and wheat had higher agreement, i.e., around $80 \%$, while others clearly require more specialist knowledge and therefore agreement was much lower. The current version of the app provided no training in land cover or land use recognition and relied only on the knowledge of the individuals taking part in the game. The main reason was to minimize the burden on players as much as possible, given that each quest involved collecting 
data according to a protocol and there is a tradeoff between how much you can ask individuals to do and the number of participants [36]. However, it is clear that some training is required if citizens are to be able to accurately classify land cover and land use. This could be in the form of some type of mapping party [37], a simple online tutorial or providing players with sample images (e.g., different types of trees or crops) in the app, which are displayed when they need to make a decision about level 2 and 3 land cover/land use. A feedback system could also be beneficial [38] so that players who have problems with the recognition of certain land cover or land use types could learn as they play the game or some type of online chat facility to ask questions about current quests could be added. Finally, the use of tools from CTA could be employed to help improve the training materials and the app more generally.

The radius of homogeneity (as specified by players in the app) and homogeneity in all directions (determined by the LUCAS photographs and satellite imagery) are clearly influencing agreement, resulting in higher values, especially for land use categories. From a remote sensing perspective, the automatic determination of homogeneous points (i.e., radius greater than $25 \mathrm{~m}$ ), especially those where several players agree, would be beneficial for both training and validating satellite imagery, in particular the most recent Sentinel data or Landsat. Thus, crowdsourcing homogeneous land cover using citizens has the potential to vastly increase the amount in-situ data available, where the lack of ground-based data has already been raised as a key issue by the EEA [39].

Another result from the analysis is that it makes no apparent difference to the expected agreement if the player is a power player or not. There are some observed differences but these are not significant once other characteristics are controlled for. When exploring other players (excluding power players), the number of observations does have an influence on agreement on level 2 land use and land cover, implying that the collective intelligence of the crowd has a positive effect [34,40]. The sliding scale of points was intended to attract players to undertake quests that had not been captured yet. Nevertheless it might be beneficial to leave the maximum point value of 100 on each point until it has been visited a minimum number of times in order to apply some type of majority voting at each location. We could also consider adding additional incentives to the game, e.g., in the style of Pokémon GO, which has demonstrated that individuals are willing to go outside and play a mobile game if the tasks are fun and challenging.

Although the campaign resulted in the collection of 2234 points at 1699 unique locations, the actual number of points used in this analysis was low. This was partly due to the fact that less than half of the points overlapped with LUCAS points, because the game was populated with eight times more points than available in LUCAS, and, of those points collected, another $50 \%$ were excluded for various reasons including lack of visibility of the location. Many of these issues have been considered, and the current version of the app, as well as the new campaign FotoQuest Go, which includes LUCAS points across Europe, have already implemented some improvements. For example, newer versions of the app now display a satellite image around the point, which helps the player to compare the surrounding area to what is seen on the imagery. This can serve as useful navigational aid, e.g., by providing alternative routes for reaching points that were previously reported as not visible or unreachable.

The 2015 LUCAS campaign took place in the spring of 2015. The data were finally released more than one year later, since the data needed further processing, i.e., transferred from a paper-based format and for quality assurance checks. A mobile application makes the data collection much easier and the data collected can be processed much faster. The results could also be made available in near real-time. Most significantly, if such gaming approaches were to become more popular and lead to the continual monitoring of land cover and land use by the public, this would provide a constant stream of information that would not only complement LUCAS data but also be useful for land cover calibration and validation. 


\section{Conclusions}

The overall agreement between the land cover and land use data at high level (L1) collected by citizens compared with authoritative data from LUCAS was close to $70 \%$. This value, although not yet high enough to be considered reliable for use in validation, is very encouraging for a first attempt at understanding the willingness of volunteers to survey data in the field and support professional surveys such as LUCAS with crowdsourced data. However, it is clear that some additional training is needed, as well as regular feedback, if citizens are to be able to distinguish between more detailed classes of land cover and land use or to improve their recognition skills over time. Moreover, detailed land use information is extremely valuable. As there was only a small difference in the overall agreement between levels 1 and 2 of the LUCAS land use classification (6\%) when compared with data from FotoQuest Austria, this may be a useful source of data for urban planning, particularly in providing information about areas with recent change. Such an app could even be used by planning authorities to direct citizens to potential areas where change is occurring to help update their databases.

The identification of land cover in homogeneous areas is the most useful from a remote sensing perspective, and this was also the easiest for players to identify with an agreement close to $80 \%$ for land cover and land use at the highest level of the classification (L1). Rather than gather land cover and land use information at heterogeneous locations, players could simply mark these spots as heterogeneous and move on. Alternatively, they could find nearby homogeneous areas and gather information at these locations instead. This would maximize the information content from the players while making it easier to recognize and classify land cover and land use.

FotoQuest Austria has been extended to Europe and will be used to gather land cover and land use data across multiple EU countries from mid-October 2016. If the app were to become more successful and be used to collect data on a regular basis, then it would provide much more up-to-date data than LUCAS data and at a potentially higher density than the current sample. Ideally, multiple quests at the same point would be undertaken in order to obtain more reliable statistics. Thus, different ways to motivate participation should be a key focus. Crowdsourcing clearly has the potential to contribute to in-situ data collection of relevance to remote sensing but there is a delicate balance between the need for rigor in the data collection protocols and the promotion of public participation, ensuring that the activity remains fun and engaging.

Acknowledgments: This work was supported by the EU FP7 funded ERC grant Crowdland (No. 617754).

Author Contributions: All of the authors contributed to the development of the idea, contributed to the running of the campaign, took part in testing the app and took part in the actual campaign. T.S. and M.K. wrote the app. J.C.L.B. analyzed the data. J.C.L.B. and L.S. wrote the paper while the remaining authors provided comments, edits and made general improvements to the manuscript.

Conflicts of Interest: The authors declare no conflicts of interest.

\section{References}

1. Clavero, M.; Revilla, E. Biodiversity data: Mine centuries-old citizen science. Nature 2014, 510, 35. [CrossRef] [PubMed]

2. Nature. Rise of the citizen scientist. Nature 2015, 524, 265.

3. Howe, J. The rise of crowdsourcing. Wired Mag. 2006, 14, 1-4.

4. Buhrmester, M.; Kwang, T.; Gosling, S.D. Amazon's Mechanical Turk: A new source of inexpensive, yet high-quality, data? Perspect. Psychol. Sci. 2011, 6, 3-5. [CrossRef] [PubMed]

5. Whittaker, J.; McLennan, B.; Handmer, J. A review of informal volunteerism in emergencies and disasters: Definition, opportunities and challenges. Int. J. Disaster Risk Reduct. 2015, 13, 358-368. [CrossRef]

6. Goodchild, M.F. Citizens as sensors: the world of volunteered geography. GeoJournal 2007, 69, $211-221$. [CrossRef]

7. Turner, A. Introduction to Neogeography; O'Reilly: Sebastopol, CA, USA, 2006. 
8. See, L.; Mooney, P.; Foody, G.; Bastin, L.; Comber, A.; Estima, J.; Fritz, S.; Kerle, N.; Jiang, B.; Laakso, M.; et al. Crowdsourcing, citizen science or Volunteered Geographic Information? The current state of crowdsourced geographic information. ISPRS Int. J. Geo-Inf. 2016, 5, 55. [CrossRef]

9. Bell, S.; Cornford, D.; Bastin, L. The state of automated amateur weather observations. Weather 2013, 68, 36-41. [CrossRef]

10. Arsanjani, J.J.; Zipf, A.; Mooney, P.; Helbich, M. (Eds.) OpenStreetMap in GIScience; Lecture Notes in Geoinformation and Cartography; Springer International Publishing: Cham, Switzerland, 2015.

11. Zook, M.; Graham, M.; Shelton, T.; Gorman, S. Volunteered Geographic Information and crowdsourcing disaster relief: A case study of the Haitian earthquake. World Med. Health Policy 2010, 2, 7-33. [CrossRef]

12. Ghosh, S.; Huyck, C.K.; Greene, M.; Gill, S.P.; Bevington, J.; Svekla, W.; DesRoches, R.; Eguchi, R.T. Crowdsourcing for rapid damage assessment: The Global Earth Observation Catastrophe Assessment Network (GEO-CAN). Earthq. Spectra 2011, 27, S179-S198. [CrossRef]

13. Connors, J.P.; Lei, S.; Kelly, M. Citizen science in the age of neogeography: Utilizing volunteered geographic information for environmental monitoring. Ann. Assoc. Am. Geogr. 2012, 102, 1267-1289. [CrossRef]

14. Conrad, C.C.; Hilchey, K.G. A review of citizen science and community-based environmental monitoring: issues and opportunities. Environ. Monit. Assess. 2010, 176, 273-291. [CrossRef] [PubMed]

15. See, L.; Fritz, S.; Dias, E.; Hendriks, E.; Mijling, B.; Snik, F.; Stammes, P.; Vescovi, F.; Zeug, G.; Mathieu, P.-P.; et al. A new generation of tools for crowdsourcing and citizen science to support Earth Observation calibration and validation. IEEE Geosci. Remote Sens. Mag. 2016, 3, 38-50. [CrossRef]

16. Fritz, S.; McCallum, I.; Schill, C.; Perger, C.; See, L.; Schepaschenko, D.; van der Velde, M.; Kraxner, F.; Obersteiner, M. Geo-Wiki: An online platform for improving global land cover. Environ. Model. Softw. 2012, 31, 110-123. [CrossRef]

17. See, L.; Fritz, S.; Perger, C.; Schill, C.; McCallum, I.; Schepaschenko, D.; Duerauer, M.; Sturn, T.; Karner, M.; Kraxner, F; et al. Harnessing the power of volunteers, the internet and Google Earth to collect and validate global spatial information using Geo-Wiki. Technol. Forecast. Soc. Chang. 2015, 98, 324-335. [CrossRef]

18. Clark, M.L.; Aide, T.M. Virtual Interpretation of Earth Web-Interface Tool (VIEW-IT) for collecting land-use/land-cover reference data. Remote Sens. 2011, 3, 601-620. [CrossRef]

19. Snik, F.; Rietjens, J.H.H.; Apituley, A.; Volten, H.; Mijling, B.; Di Noia, A.; Heikamp, S.; Heinsbroek, R.C.; Hasekamp, O.P.; Smit, J.M.; et al. 3187 iSPEX citizen scientists Mapping atmospheric aerosols with a citizen science network of smartphone spectropolarimeters. Geophys. Res. Lett. 2014, 41. [CrossRef]

20. Eurostat. LUCAS 2015 (Land Use/Cover Area Frame Survey). Technical Reference Document C1 Instructions for Surveyors; Eurostat: Luxembourg, 2015.

21. Eurostat. Task Force Meeting: LUCAS2018 and Beyond; Reference Paper; Eurostat: Luxembourg, 2015.

22. EEA. The Thematic Accuracy of Corine Land Cover 2000-Assessment Using LUCAS; European Environment Agency: Copenhagen, Denmark, 2006.

23. Büttner, G.; Eiselt, B. LUCAS and CORINE Land Cover; EEA and Eurostat: Luxembourg, 2013.

24. Gallego, F.J. Validation of GIS layers in the EU: Getting adapted to available reference data. Int. J. Digit. Earth 2011, 4, 42-57. [CrossRef]

25. Gallego, F.J.; Palmieri, A.; Ramos, H. Sampling System for LUCAS 2015; EUR 27595 EN; Joint Research Centre of the European Commission: Ispra, Italy, 2015.

26. Eurostat. LUCAS 2015 Technical Reference Document C3 Classification (Land Cover E Land Use); Eurostat: Luxembourg, 2015.

27. Deterding, S.; Sicart, M.; Nacke, L.; O'Hara, K.; Dixon, D. Gamification using game-design elements in non-gaming contexts. In CHI '11 Extended Abstracts on Human Factors in Computing Systems; ACM Press: New York, NY, USA, 2011; pp. 2425-2428.

28. Bowser, A.; Hansen, D.; Preece, J. Gamifying citizen science: Lessons and future directions. In Proceedings of the CHI 2013: Designing Gameification: Creating Gameful and Playful Experiences, Paris, France, 27 April-2 May 2013.

29. See, L.; Sturn, T.; Perger, C.; Fritz, S.; McCallum, I.; Salk, C. Cropland Capture: A gaming approach to improve global land cover. In Proceedings of the AGILE'2014 International Conference on Geographic Information Science, Castellon, Spain, 3-16 June 2014. 
30. Sturn, T.; Wimmer, M.; Salk, C.; Perger, C.; See, L.; Fritz, S. Cropland Capture-A game for improving global cropland maps. In Proceedings of the Foundation of Digital Games (FDG 2015), Pacific Grove, CA, USA, 20-22 June 2015.

31. Eurostat Overview of LUCAS. Available online: http://ec.europa.eu/eurostat/web/lucas/overview (accessed on 2 August 2016).

32. Alonso, K.; Espinoza-Molina, D.; Datcu, M. LUCAS Visual Browser: A tool for land cover visual analytics. In Proceedings of the 2015 IEEE International Geoscience and Remote Sensing Symposium (IGARSS), Milan, Italy, 26-31 July 2015; pp. 1484-1487.

33. Burnham, K.P.; Anderson, D.R. Model Selection and Multimodel Inference: A Practical Information-Theoretic Approach, 2nd ed.; Springer: New York, NY, USA, 2002.

34. Haklay, M.; Basiouka, S.; Antoniou, V.; Ather, A. How many volunteers does it take to map an area well? The validity of Linus' Law to volunteered geographic information. Cartogr. J. 2010, 47, 315-322. [CrossRef]

35. Kerle, N.; Hoffman, R.R. Collaborative damage mapping for emergency response: The role of Cognitive Systems Engineering. Nat. Hazards Earth Syst. Sci. 2013, 13, 97-113. [CrossRef]

36. Tweddle, J.C.; Robinson, L.D.; Pocock, M.J.O.; Roy, H. Guide to Citizen Science: Developing, Implementing and Evaluating Citizen Science to Study Biodiversity and the Environment in the UK; Natural History Museum and NERC Centre for Ecology \& Hydrology: Wallingford, UK, 2012.

37. Mooney, P.; Minghini, M.; Stanley-Jones, F. Observations on an OpenStreetMap mapping party organised as a social event during an open source GIS conference. Int. J. Spat. Data Infract. Res. 2015, 10, 138-150.

38. Pocock, M.J.O.; Chapman, D.S.; Sheppard, L.J.; Roy, H.E. Choosing and Using Citizen Science: A Guide to When and How to Use Citizen Science to Monitor Biodiversity and the Environment; NERC Centre for Ecology \& Hydrology: Wallingford, UK, 2014.

39. European Environment Agency. Montoring Matters: In-Situ Coordinaton in Support of Copernicus Operations; European Environment Agency: Copenhagen, Denmark, 2013.

40. Surowiecki, J. The Wisdom of Crowds; Anchor Books: New York, NY, USA, 2005.

(C) 2016 by the authors; licensee MDPI, Basel, Switzerland. This article is an open access article distributed under the terms and conditions of the Creative Commons Attribution (CC-BY) license (http://creativecommons.org/licenses/by/4.0/). 